\title{
Analysis of Entropy Generation Minimization in Circular Porous Fins
}

\author{
Seyfolah Saedodin, ${ }^{1}$ Siamak Sadeghi, ${ }^{2}$ and Majid Shahbabaei ${ }^{2}$ \\ ${ }^{1}$ Science and Research Branch, Department of Mechanical Engineering, Islamic Azad University, P.O. Box 35131-3711, Semnan, Iran \\ ${ }^{2}$ Department of Mechanical Engineering, Islamic Azad university, Semnan Branch, P.O. Box 35131-3711, Semnan, Iran
}

Correspondence should be addressed to Siamak Sadeghi, siamak.sadeghi1986@gmail.com

Received 27 July 2012; Accepted 5 September 2012

Academic Editors: A. Gil and A. M. Seayad

Copyright (C) 2012 Seyfolah Saedodin et al. This is an open access article distributed under the Creative Commons Attribution License, which permits unrestricted use, distribution, and reproduction in any medium, provided the original work is properly cited.

This work introduces a simple method of exergy analysis in a typical circular porous fin. The entropy generation of any thermodynamic system provides a useful measure of the extent of irreversibility. The irreversibility causes the loss of useful work (exergy) in the system and hence the loss of exergy has to be minimized. Entropy generation is a parameter that quantifies the loss of exergy. Circular fins are relatively good heat transfer augmentation features with superior aerodynamic performance and as a result find application in some solar air heaters. In this paper, the entropy generation in a circular porous fin is calculated and its performance is compared with respect to entropy generation. Also shown in porous fins, with increase of porosity. The entropy generation number $N_{s}$ will increase; also states with porosity have higher entropy generation number than $N_{s}$ states with nonporosity $(\varphi=0)$. Also at higher Reynolds number the effect of the $\operatorname{Re}_{L}$ on $N_{s} T$ is negligible, but at lower Reynolds number the variation of the $N_{s} P$ is negligible. Also we can see that with increased porosity $(\varphi)$, the entropy generation $\left(S_{\text {gen }}\right)$ will decrease. The entropy generation is calculated for circular porous fins with mass constraint.

\section{Introduction}

The term extended surfaceis used to describe a system in which the area of a surface is increased by the attachment of fins. A fin accommodates energy transfer by conduction within its boundaries, while its exposed surfaces transfer energy to the surroundings by convection or radiation or both. Fins are commonly used to augment heat transfer from electronic components, automobile radiators, engine and compressor cylinders, control devices, and a host of other applications. Fins are used to enhance convective heat transfer in a wide range of engineering applications and offer a practical means for achieving a large total heat transfer surface area without the use of an excessive amount of primary surface area. Fins are commonly applied for heat management in electrical appliances such as computer power supplies or substation transformers. Other applications include IC engine cooling, such as fins in a car radiator. Heat and mass transfer through saturated porous media is an important development and an area of very rapid growth in contemporary heat transfer research. Although the mechanics of fluid flow through porous media has preoccupied engineers and physicists for more than a century, the study of heat transfer has reached the status of a separate field of research during the last three decades [1]. The description of heat and fluid flow through a porous medium saturated with fluid (liquid or gas) is based on a series of special concepts that are not found in the pure-fluid heat transfer. Examples are the porosity and the permeability of the porous medium and the volume-averaged properties of the fluid flowing through the porous medium. The porosity of the porous medium is defined as

$$
\varphi=\frac{\text { void volume contained in porous medium sample }}{\text { total volume of porous medium sample }} \text {. }
$$

The increased awareness that the world's energy resources are limited has caused many countries to reexamine their energy policies and take drastic measures in eliminating waste. It has also sparked interest in the scientific community 
to take a closer look at the energy conversion devices and to develop new techniques to better utilize the existing limited resources. The first law of thermodynamics deals with the quantity of energy and asserts that energy cannot be created or destroyed. This law merely serves as a necessary tool for the bookkeeping of energy during a process and offers no challenges to the engineer. The second law, however, deals with the quality of energy. More specifically, it is concerned with the degradation of energy during a process, the entropy generation, and the lost opportunities to do work. The second law of thermodynamics has proved to be a very powerful tool in the optimization of complex thermodynamic systems. The second law of thermodynamics uses an exergy balance for the analysis and the design of thermal systems. The importance of this thermal design technique in the general area of heat transfer augmentation and energy conservation is fully recognized by the heat transfer community $[2,3]$. The traditional approach to the optimization of fins consists of minimizing the consumption of the fin material for the execution of a specified heat transfer task. More than a half-century ago, Schmidt stated intuitively that a two-dimensional fin must have a paraboliclaw based cross-sectional profile if it is to require the least material (volume) for a certain heat transfer rate, and this design principle was later proved by [4] who worked on the formulation of variational calculus [5]. This design principle has been steadily brought closer to the realities of fin manufacturing and heat exchanger operation by a number of contributors who have analyzed the role of radiation, twodirectional heat transfer (curvature) temperature-dependent thermal conductivity and variable heat transfer coefficient [6-10]. Entropy generation in a thermodynamics system is defined as the difference between the entropy change of the system and the net entropy transport into the system and is a path function of the thermodynamics system. According to the second law of thermodynamics, the entropy change of a system is always greater than or equal to the net entropy transport into the system. The first and second laws of thermodynamics, taken together, state that the entropy generated by any engineering system is proportional to the work lost (destroyed) irreversibly by the system. Truth is expressed concisely as the Gouy-Stodola theorem [11]:

$$
W_{\text {lost }}=T_{0} \sum_{\text {total }} S_{\text {gen }},
$$

where $W_{\text {lost }}$ is the lost available work (lost exergy) [12], $T_{0}$ is the absolute temperature of the environment, and $S_{\text {gen }}$ is the entropy generated in each compartment of the system. Equation (2) implies the thermodynamics irreversibility (entropy generation) of each system component contributes to the aggregate loss of available work in the system ( $\left.W_{\text {lost }}\right)$. Circular fins serve as components in a wide range of heat transfer applications. Therefore, in order to conserve available work (exergy), it is necessary to approach the design of such fins from the point of view of entropy generation minimization. The trade-off between heat transfer and fluid friction is a classical dilemma in heat exchanger design $[3,5]$. The irreversibility minimization philosophy places trade-off on a solid foundation, as heat transfer and fluid

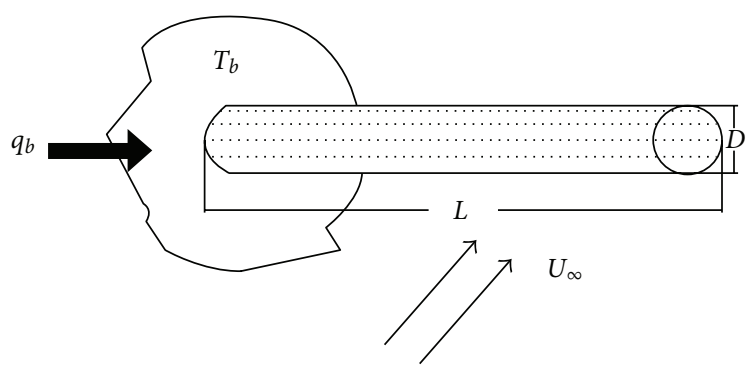

Figure 1: Porous fin nomenclature.

drag are both mechanisms for entropy generation. Using the bulk level of entropy production, [13] found the optimal design of radial fin geometry with respect to Reynolds number in both laminar and turbulent convection. [14] showed a numerical code in order to evaluate the effects of different parameters of combustion in porous media, that the governing equations including Navier-stokes, the solid and gas energy and the chemical species transport equations are solved using a multistep reduced kinetic mechanism. In [15], EGM (entropy generation minimization) approach is implemented for the analysis of optimal thermal mixing and temperature uniformity due to natural convection in square cavities filled with porous medium for the material processing application.

\section{Materials and Methods}

2.1. Analysis Using the First and Second Laws of Thermodynamics. The entropy generated by a single porous fin in cross-flow can be evaluated based on the general model presented in Figure 1. A circular porous fin of arbitrary crosssection is suspended in a uniform stream with velocity $U_{\infty}$, temperature $T_{\infty}$, and solar radiation $q_{r}$. The heat transfer $q_{b}$ is driven by the temperature difference between the fin base $T_{b}$ and the free stream, $T_{\infty}$. In addition, the cross-flow arrangement is responsible for a net drag force $F_{D}$, which is transmitted through the porous fin. At steady state, by using first and second laws of thermodynamics we have:

$$
\begin{gathered}
q_{b}+q_{r}-q_{\text {fluid }}+F_{D} U_{\infty}=0, \\
S_{\text {gen }}=\frac{q_{\text {fluid }}}{T_{\infty}}-\frac{q_{b}}{T_{b}}-\frac{q_{r}}{T_{\infty}} \geq 0 .
\end{gathered}
$$

The following assumptions are made to solve this problem.

(i) The porous medium is isotropic and homogenous.

(ii) The porous medium is saturated with single-phase fluid.

(iii) Physical properties of both fluid and solid matrix are constant.

(iv) The temperature inside fin is only function of $x$ and velocity inside fin is very low.

(v) There is no temperature variation across the fin thickness.

(vi) The solid matrix and fluid are assumed to be at local thermal equilibrium with each other. 
(vii) The interactions between the porous medium and the clear fluid can be simulated by the Darcy formulation.

(viii) All conditions are the same as flow around a solid fin and only variable parameter is porosity.

2.2. Circular Porous Fin. Circular fin is one of the simplest geometries, since the heat transfer and drag force depend on only two dimensions, the length $L$ and the diameter of the circular cross-section $D$. In (3) and (4), $q_{b}$ is the amount of heat which is transferred from the base wall to the fin, $q_{\text {fluid }}$ is the amount of heat, which is transferred from porous fin to the rest of the fluid, and $q_{r}$ is the amount of heat, which is transferred from solar to porous fin. The free stream temperature and free stream velocity are taken as $T_{\infty}$ and $U_{\infty}$, respectively. The entropy generation in the heat transfer and fluid flow arrangement is $S_{\text {gen }}$. By combining (3) and (4), $q_{b}$ and $q_{r}$ can be eliminated and the following equation is obtained:

$$
S_{\text {gen }}=\frac{q_{b}}{T_{\infty}\left[1+T_{\infty} / \theta_{b}\right]}+\frac{F_{D} U_{\infty}}{T_{\infty}} .
$$

The relationship between the fin base heat flux and the steam temperature difference is (Kern 1980)

$$
\theta_{b}=\frac{q_{b}}{(\pi / 4) K_{f} D^{2} m \tanh (m l)} .
$$

With the specification of $S_{\text {gen }}$ and $q_{b}$ relations essentially, the entropy generation number $N_{s}$ associated with the fin for design purposes can be determined by [16]:

$$
N_{s}=\frac{S_{\text {gen }}}{\left(q_{b}^{2} U_{\infty} / k \nu T_{\infty}^{2}\right)}
$$

From the Darcy's model for porous media, we have

$$
U_{\infty}=\frac{g k \beta}{\nu\left(T_{b}-T_{\infty}\right)} .
$$

Also the effective thermal conductivity of the porous fin is given by

$$
K_{\mathrm{eff}}=\varphi k_{f}+(1-\varphi) k_{s}
$$

Substitution of (5) and (6) into (7) gives

$$
N_{s}=\frac{1}{\left(K_{r}\right)(\pi / 2)\left(\operatorname{Re}_{L}\right)(\lambda)\left(T / T_{\infty}\right)+(\pi / 2)\left(\operatorname{Re}_{L}\right)(\mathrm{Nu})^{0.5}\left(K_{r}\right)\left(K_{f}\right)^{2} \tanh \left[2(\mathrm{Nu})^{0.5}\left(1 / K_{r}\right)^{0.5}\right]}+B\left(\operatorname{Re}_{L}\right)^{2}\left(\frac{1}{K_{r}}\right)^{2}\left(\frac{1}{\lambda}\right)^{2}\left(C_{D}\right)
$$

Or

$$
N_{s}=N_{s} T+N_{s} P \text {, }
$$

where $K_{r}=K_{\text {eff }} / K_{f}$, the dimensionless number, is the ratio of porous fin and fluid thermal conductivities. The slenderness ratio $(\lambda=1 / L)$ is defined as the ratio between the length and characteristic dimension of the circular fins and $B$ is a constant value. The equation for entropy generation number for circular porous fin is given by (10) respectively. In this equation, the first item indicates the entropy generation number due to temperature difference, $N_{s} T$, but the second item indicates $N_{s} P$. The Nusselt number and drag coefficient can be evaluated from the results developed for a single circular cylinder in cross flow and are given by (13a) and (13b). From [17] one has:

$$
B=\frac{\rho \nu^{3} T_{\infty}}{\pi D^{3}\left(T_{b}-T_{\infty}\right)^{2}}
$$

$$
\begin{gathered}
1 \leq \mathrm{Re}_{L} \leq 40, \quad \mathrm{Nu}=0.657 \mathrm{Re}_{L}{ }^{0.4}, \\
40 \leq \mathrm{Re}_{L} \leq 1 * 10^{3}, \quad \mathrm{Nu}=0.447 \mathrm{Re}_{L}^{0.5}, \\
1 * 10^{3} \leq \mathrm{Re}_{L} \leq 2 * 10^{5}, \quad \mathrm{Nu}=0.228 \mathrm{Re}_{L}^{0.6}, \\
2 * 10^{5} \leq \mathrm{Re}_{L} \leq 1 * 10^{6}, \quad \mathrm{Nu}=0.067 \mathrm{Re}_{L}^{0.7} .
\end{gathered}
$$

From [18], one has

$$
\begin{gathered}
1 \leq \operatorname{Re}_{L} \leq 4, \quad C_{D}=10 \operatorname{Re}_{L}^{-0.6}, \\
4 \leq \operatorname{Re}_{L} \leq 4 * 10^{3}, \quad C_{D}=5.484 \mathrm{Re}_{L}{ }^{-0.246}, \\
4 * 10^{3} \leq \operatorname{Re}_{L} \leq 2 * 10^{5}, \quad C_{D}=1.1 .
\end{gathered}
$$

2.3. Optimization. The first step to optimize a thermodynamic system is to determine a proper objective function. The next step is to minimize this objective function which can be done using a number of different numerical, analytical and graphical methods. Using the equation presented in, Section 2.2, the objective function is optimized. Here, the slenderness ratio is chosen as objective function. Minimization of $N_{s}$ with respect to $\lambda$ is achieved in a straightforward manner by solving (12) as follows:

$$
\frac{\partial N_{s}}{\partial \lambda}=0
$$

\section{Results}

The engineering significance of (10) is that the optimum fin length can be calculated immediately, provided ReL is specified. Substituting (10) into (8), it is possible to obtain the minimum $N_{s}$ for circular porous fins, corresponding to optimum pin length, $N_{s}\left(\lambda_{\text {opt }}, \operatorname{Re}_{L}\right)$ as shown in Figure 2. The optimum slenderness ratio of the circular porous fin to produce minimum entropy generation number various values of $\operatorname{Re}_{L}$. 


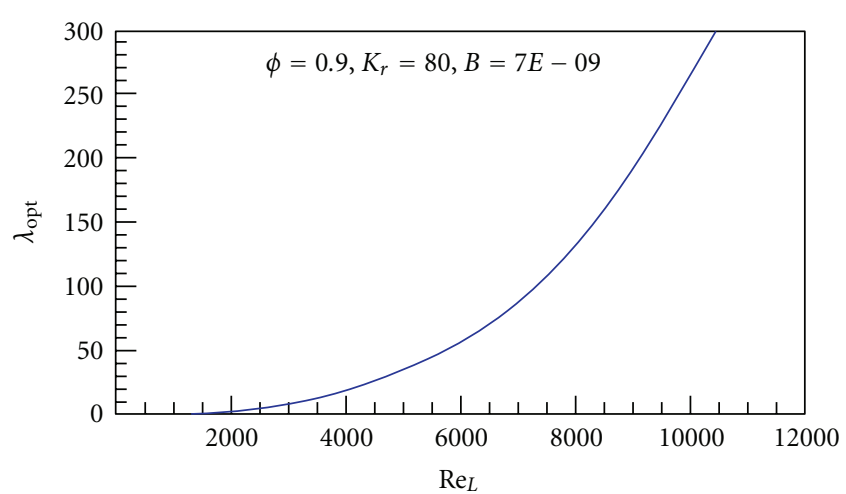

FIGURE 2: Optimum slenderness ratio of the circular porous fin for minimum entropy.

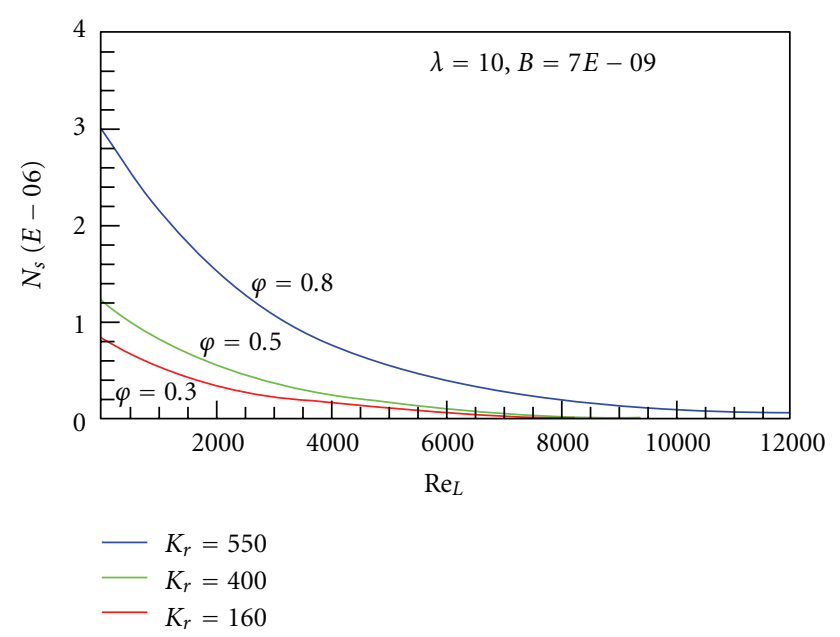

FIGURE 3: Variation of entropy generation number with Reynolds number of circular porous fin with variation of values $\varphi$.

Figure 3 shows the entropy generation number $N_{s}$ for circular porous fin with the variation of values of $\varphi$ and $\operatorname{Re}_{L}$. The values of $\varphi$ were varied between $0.3,0.5$, and 0.8 , while keeping $\lambda=10$. From Figure 3, we can see that by increasing porosity $\varphi$, in low Reynolds number, the entropy generation number $N_{s}$ will increase. But in high Reynolds number, the entropy generation due to the fluid friction is approximately constant.

Figure 4 shows comparison between fins with porosity and without porosity. It is observed that in fins with porosity $(\varphi=0.9)$, the entropy generation number is more than without porosity $(\varphi=0)$. Also in the state of $\varphi=0.9$, in high Reynolds due to the fluid friction, $N_{s}$ will increase.

In Figures 5 and 6, the variation of $N_{s} T$ and $N_{s} P$ with respect to the characteristic Reynolds number for the circular porous fins is presented. At high Reynolds number the effect of the $\operatorname{Re}_{L}$ on $N_{s} T$ is negligible and the values of the $N_{s} P$ at lower Reynolds number are negligible.

Figure 7 shows variation entropy generation $\left(S_{\text {gen }}\right)$ with Reynolds number and variable values of $\varphi, K_{r}$. From Figure 7 we can see that with increased that porosity $(\varphi)$, the entropy generation will decrease. Also with the increase of Reynolds

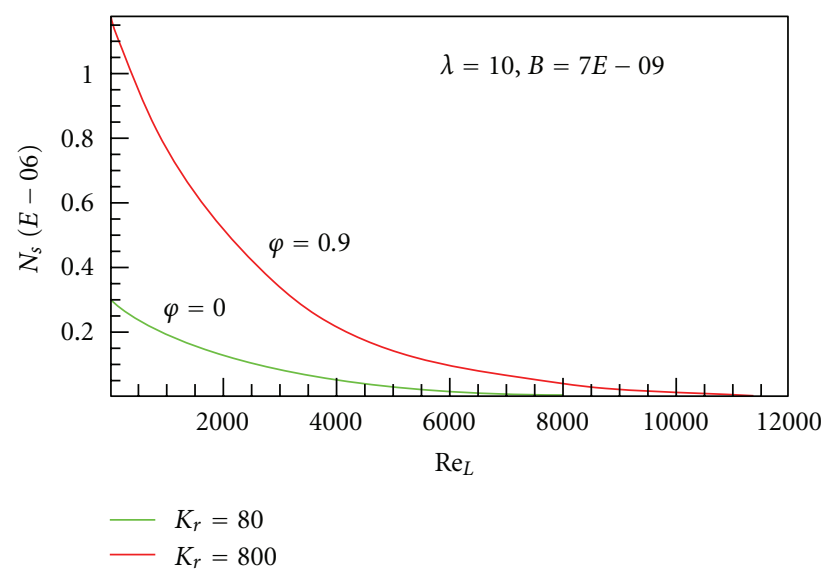

FIgure 4: Comparison between fins with porosity and without porosity.

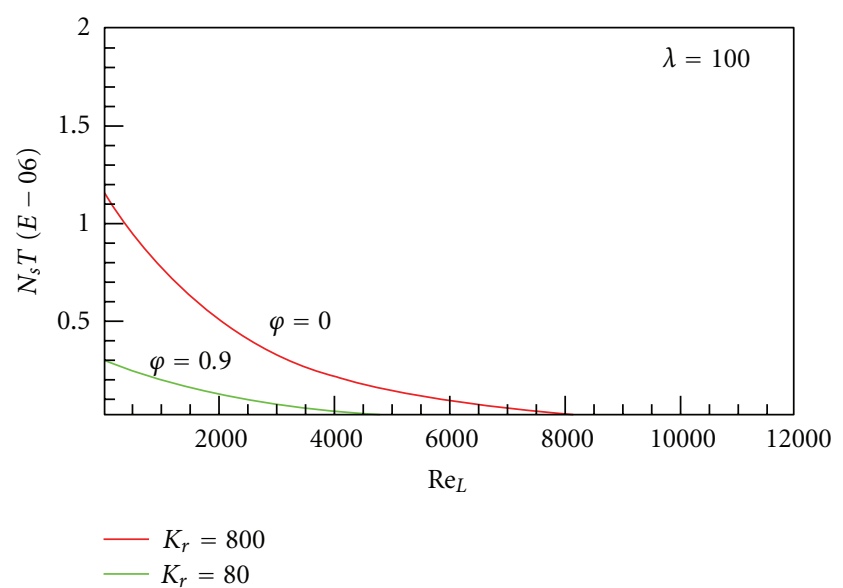

Figure 5: Variation of $N_{s} T$ with Reynolds number and $\varphi, K_{r}$.

number, the values of entropy generation $\left(S_{\text {gen }}\right)$ at all them, will increase.

\section{Discussion and Conclusion}

A quiet revolution is taking place in thermodynamics and it amounts to the closing of the gap between thermodynamics and heat transfer. The method and field that unite these classical disciplines is entropy generation minimization. Today EGM is an established method in both fundamental and applied heat transfer. This work introduces a simple method of analysis to study exergy in porous fin. By using the first and second laws of thermodynamics we will contribute to entropy generation. The entropy generation of any thermodynamics system provides a useful measure of the extent of irreversibility. Entropy generation is one parameter that quantifies the loss of exergy. In this paper, the entropy generation of circular porous fin in cross-flow of air and radiation is calculated and their performances are compared with respect to entropy generation. This paper shows that the entropy generation number $N_{s}$ has a clear minimum with respect to Reynolds number $\mathrm{Re}_{L}$. In porous fins, with 


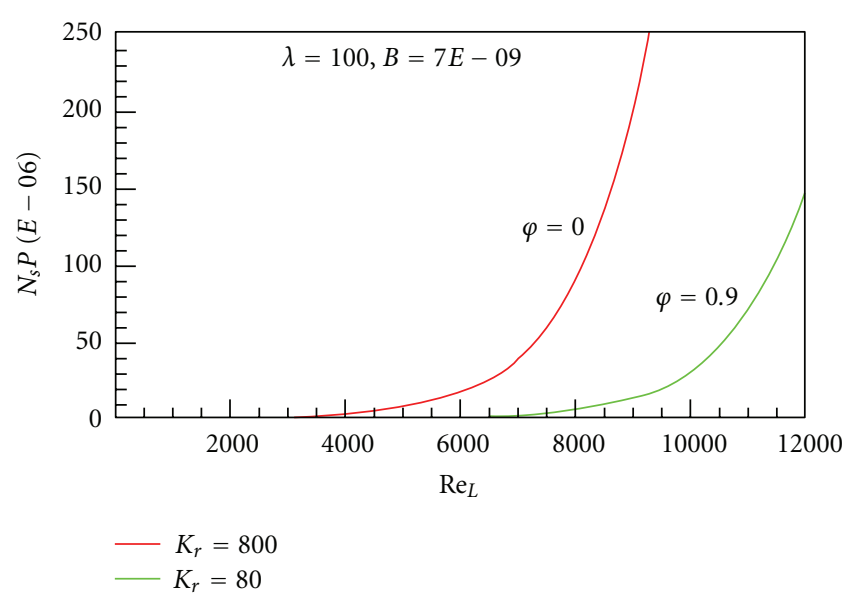

Figure 6: Variation of $N_{s} P$ with Reynolds number and $\varphi, K_{r}$.

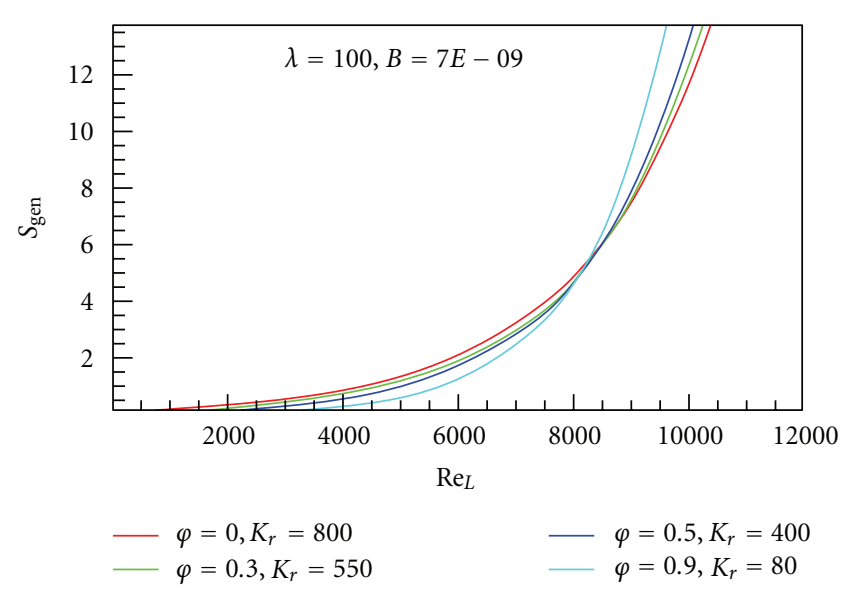

Figure 7: Variation of $S_{\text {gen }}$ with Reynolds number and $\varphi, K_{r}$.

increase of porosity, the entropy generation number $N_{s}$ will increase, also states of porosity have higher entropy generation number $N_{s}$ than states of non-porosity. Also at higher Reynolds number, the effect of the $\operatorname{Re}_{L}$ on $N_{s} T$ is negligible, but at lower Reynolds number the variation of the $N_{s} P$ is negligible. Also we can see that with increased porosity $(\varphi)$, the entropy generation $\left(S_{\text {gen }}\right)$ will decrease.

\section{Nomenclature}

A : Cross-sectional area of the fins in $\mathrm{m}$

$D$ : Diameter of the circular cross-section

$F_{D}$ : Drag force exerted by the cross-flow over the fin in $\mathrm{N}$

$k$ : Thermal conductivity of the working fluid in $\mathrm{W} / \mathrm{m} \cdot \mathrm{K}$

L: Characteristic dimension of the fins, $D$ for circular fins

$l$ : Length of the fins in $\mathrm{m}$

$K_{r}$ : Thermal conductivity ratio, $\left(k_{\text {eff }} / k_{f}\right)$

$N_{s}$ : Entropy generation number

$\theta b$ : Base temperature difference, $\left(T_{b}-T_{\infty}\right)$ $q_{b}: \quad$ Heat transfer through the base of the fin in $\mathrm{w}$

$q$ : Heat transfer to the free stream in $\mathrm{w}$

$S_{\text {gen }}:$ Entropy generation in $\mathrm{W} / \mathrm{K}$

$T: \quad$ Free stream temperature in $\mathrm{K}$

$T_{b}: \quad$ Base fin temperature in $\mathrm{K}$

$U_{\infty}$ : Free stream velocity in $\mathrm{m} / \mathrm{sec}$

$W_{\text {lost }}$ : Power lost due to the irreversibility in $\mathrm{W}$

$v: \quad$ Kinematic viscosity

$\rho: \quad$ Density

$\Phi: \quad$ Porosity

$\beta$ : $\quad$ Coefficient of volumetric thermal expansion

g: $\quad$ Gravity constant

$\mathrm{Re}_{L}$ : Reynolds number in Length of fin

$N_{s} T$ : Entropy generation number by Temperature difference

$N_{s} P: \quad$ Entropy generation number by Drag force.

\section{Subscripts}

s: Solid properties

$f$ : Fluid properties

eff: Porous properties.

\section{References}

[1] D. A. Nield and A. Bejan, Convection in Porous Media, Springer, New York, NY, USA, 2nd edition, 1999.

[2] A. E. Bergles, R. L. Webb, G. H. Junkhan, and Jensen, "Bibliography on augmentation of convective heat and mass transfer," Report HTL-19 ISU-ERI-AMES-79206, lowa State University, 1979.

[3] G. H. Junkhan, A. E. Bergles, and R. L. Webb, "Research workshop on energy conservation through enhanced heat transfer," Report HTL-21 ISU-ERI-AMES-80063, lowa State University, 1979.

[4] R. J. Duffin, "A variational problem relating to cooling fins," Journal of Mathematics and Mechanics, vol. 8, pp. 47-56, 1959.

[5] D. Poulikakos and A. Bejan, "Fin geometry for minimum entropy generation in forced convection," Transactions of the ASME, vol. 104, no. 4, pp. 616-623, 1982.

[6] C. J. Maday, "The minimum weight one-dimensional straight fin," ASME Journal of Engineering for Industry, vol. 96, no. 1, pp. 161-165, 1974.

[7] S. Guceri and C. J. Maday, "A least weight circular cooling fin," ASME Journal of Engineering for Industry, vol. 97, no. 4, pp. 1190-1193, 1975.

[8] P. Razeols and K. Imre, "The optimum dimensions of circular fins with variable thermal parameters," ASME Journal of Heat Transfer, vol. 102, pp. 420-425, 1980.

[9] D. Q. Kern and A. D. Kraus, Extended Surface Heat Transfer, McGraw-Hill, New York, NY, USA, 1980.

[10] A. D. Kraus and A. D. Snider, "New parameterizations for heat transfer in fins and spines," ASME Journal of Heat Transfer, vol. 102, no. 3, pp. 415-419, 1980.

[11] J. Szargut, "International progress in second law analysis," Energy, vol. 5, no. 8-9, pp. 709-718, 1980.

[12] J. Kestin, "Availability: the concept and associated terminology," Energy, vol. 5, no. 8-9, pp. 679-692, 1980.

[13] B. N. Taufiq, H. H. Masjuki, T. M. I. Mahlia, R. Saidur, M. S. Faizul, and E. Niza Mohamad, "Second law analysis for optimal thermal design of radial fin geometry by convection," 
Applied Thermal Engineering, vol. 27, no. 8-9, pp. 1363-1370, 2007.

[14] M. Bidi, M. R. H. Nobari, and M. S. Avval, "A numerical evaluation of combustion in porous media by EGM (Entropy Generation Minimization)," Energy, vol. 35, no. 8, pp. 34833500, 2010.

[15] R. S. Kaluri and T. Basak, "Entropy generation due to natural convection in discretely heated porous square cavities," Energy, vol. 36, no. 8, pp. 5065-5080, 2011.

[16] A. Bejan, Entropy Generation Minimization, CRC Press, New York, NY, USA, 1996.

[17] A. Žukauskas, "Heat transfer from tubes in crossflow," Advances in Heat Transfer, vol. 8, pp. 93-160, 1972.

[18] B. Gebhart, Heat Transfer, McGraw-Hill, New York, NY, USA, 1971. 

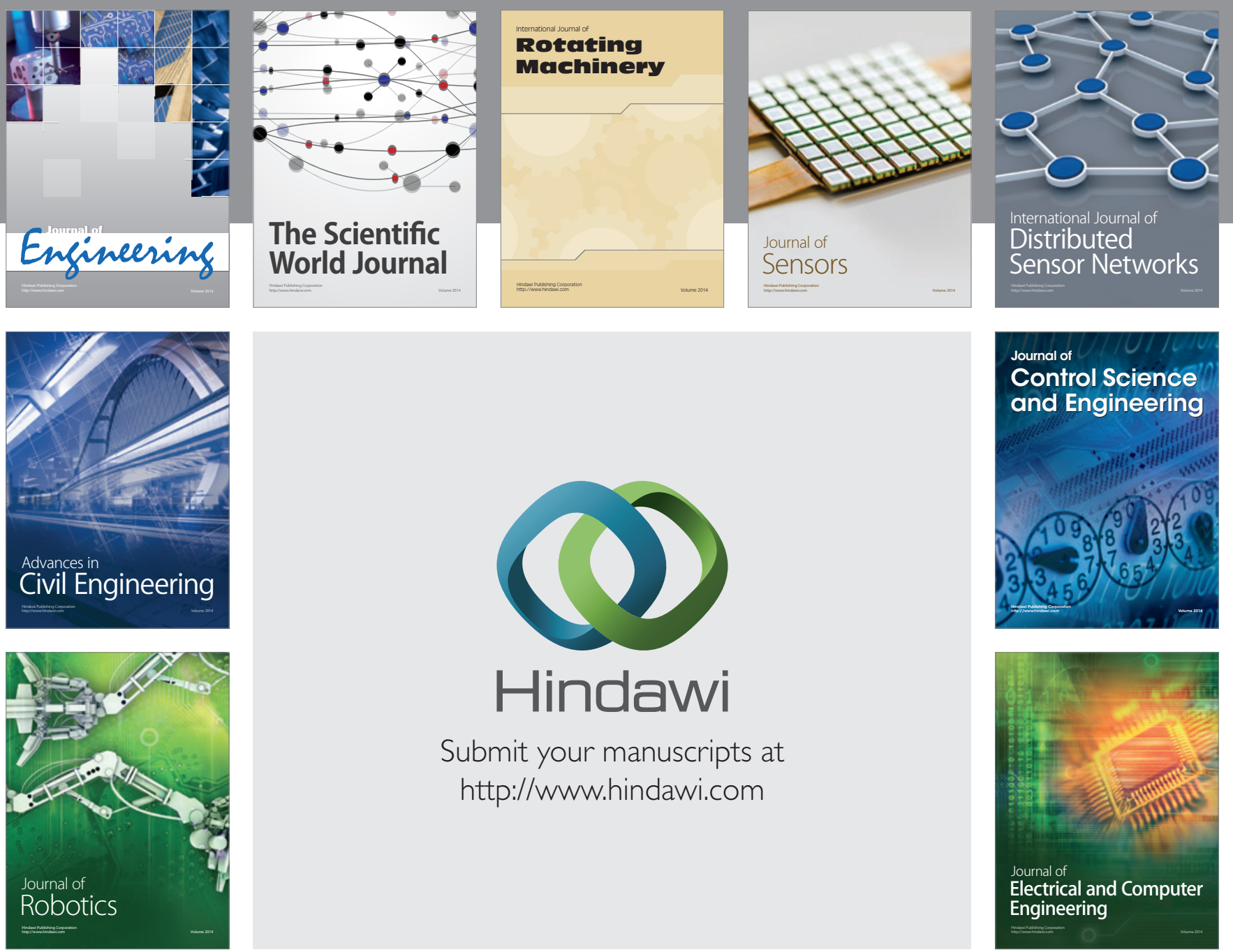

Submit your manuscripts at

http://www.hindawi.com
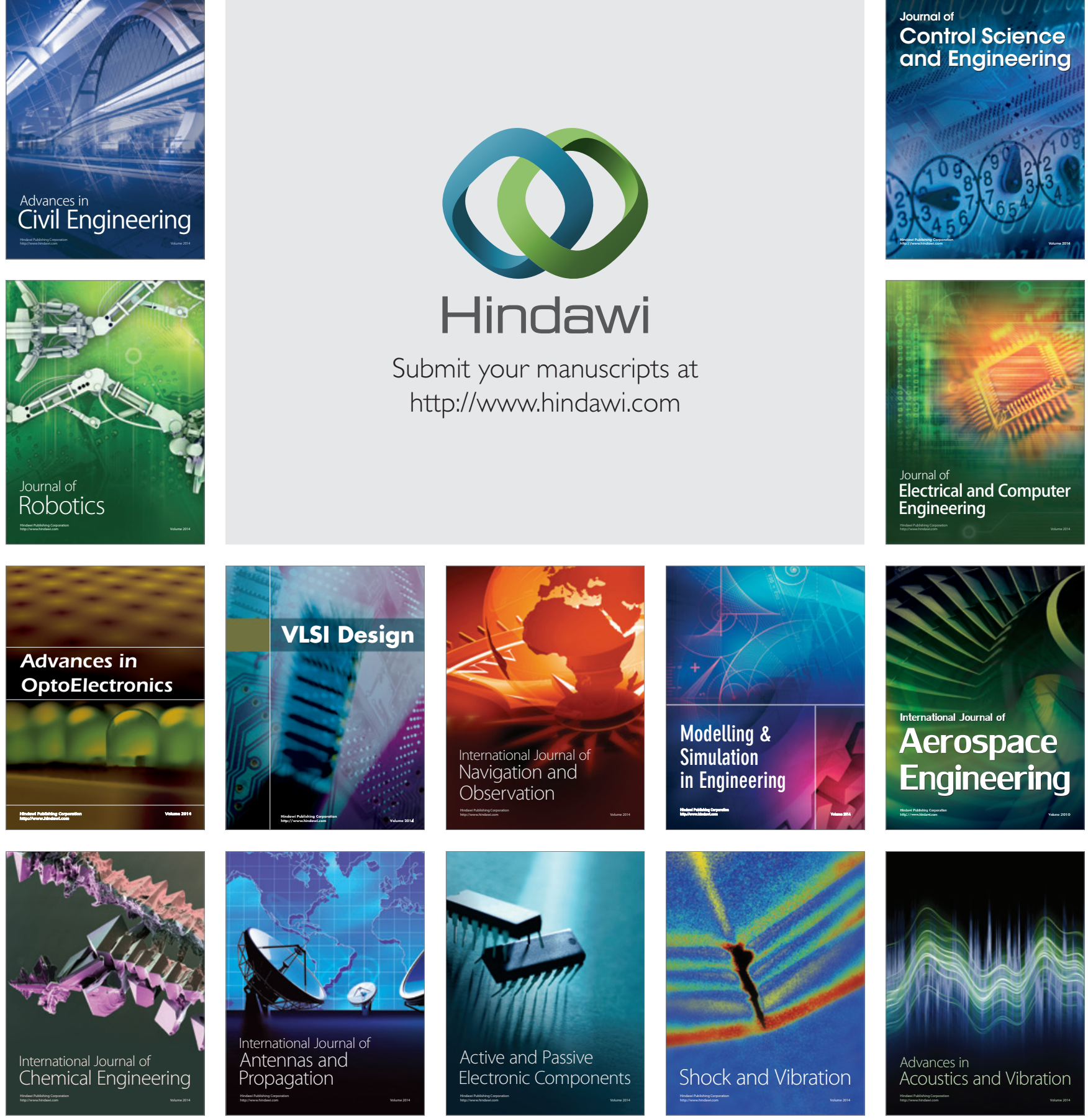